\title{
Efficient Classification of Breast Lesion based on Deep Learning Technique
}

\author{
Bandita Sen and V. Vedanarayanan
}

\begin{abstract}
In this paper, a new method for Classification of Breast Cancer Images using deep Learning Algorithm are proposed. The Classification Algorithm appears as various features extracted from Healthy Breast images and Unhealthy Breast images. The segmentation algorithm is Deep learning and then Classification Tool is ANN [Artificial Neural Network]. Algorithms are performed and different features are extracted. The features are Breast Image Mean, Breast Image Standard Deviation, Breast image Peak Signal to Noise Ratio [PSNR], breast image Contrast to Noise Ratio [CNR]. ANN tool showing breast image results.
\end{abstract}

Keywords--- Deep Learning, Greedy Algorithm, Morphological Operators, Breast Database, ANN Classification Tool

\section{INTRODUCTION}

$\mathrm{O}$ UR Work in this paper mainly Deep learning Based Breast cancer segmentation using breast cancer[Calcification] Images, also classify the breast images using ANN tool. The Classification performs by using the features which is extracted from breast images. The features are Mean, Standard Deviation, SNR [signal to noise ratio], CNR [contrast to noise ratio].

In section II explains over all work flow of deep learning algorithm. The Section III \& IV analyze the filtering and enhancement algorithm. Section V explains a Morphological performance and section VI Analyze the ANN tool performance.

\section{DEEP LEARNING ALgORITHM}

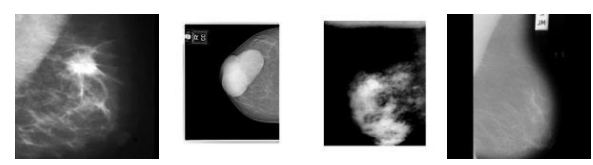

Figure 1: Breast Database

Deep Learning Algorithm methods are an evolution of conventional neural network [1],[2]. These approaches generally involve various non-linear transformations of the data with the objective of contribution to abstracts and appropriate representations [3]. These methods are becoming popular, generally the exceeding traditional approaches includes handcrafted features for data representation and machine learning methods are used for learning task [4],[5].

Bandita Sen, ME (Applied Electronics), Sathyabama University, Chennai, India.E-mail:bandita.jisce@gmail.com

V. Vedanarayanan, Assistant Professor, ETCE Department, Sathyabama University, Chennai, India.E-mail:veda_gopal@rediffmail.com

DOI: 10.9756/BIJAIP.10446

\section{Method of Pixel Points}

One of the advance algorithms in unsupervised deep learning is the method of pixel points. In the method of pixel point, the unknown parameters (of pixels) in the model are related to the pixel points of one or more casual pixel points, and thus, these unknown parameters can be predicted given the pixel points. The pixel points are mostly predicted from breast regions in an observed way. The basic pixel points are first and second order pixel points for a random pixel points, the first order pixel points is the mean pixels, and the second order pixel points is the covariance matrix (when the mean is zero). Higher order pixel points are mostly represented using tensors which are the generalization of matrices to higher orders as multi-dimensional pixels.

\section{Method of Descriptors Analysis}

The method is to estimate the $k$ unknown pixels $\theta_{1}, \theta_{2}, \ldots, \theta_{k}$ describe the diffusion $f_{w}(\omega ; \theta)$ of the random pixels or variables $W$. Assume the first $k$ pixel points of the true diffusion can be expressed as function $\theta^{s:}$

$$
\begin{aligned}
& \mu_{1} \equiv E[W]=g_{1}\left(\theta_{1}, \theta_{2}, \ldots, \theta_{k}\right) \\
& \mu_{2} \equiv E\left[W^{2}\right]=g_{2}\left(\theta_{1}, \ldots, \theta_{k}\right) \\
& \mu_{k} \equiv E\left[W^{k}\right]=g_{2}\left(\theta_{1}, \ldots, \theta_{k}\right)
\end{aligned}
$$

Surmise Breast image region pixel region $n$ is pinched, resulting in the values $\omega_{1}, \ldots, \omega_{n}$ for $j=1, \ldots, k$ let

$$
\mu_{j}=\frac{1}{n}_{i=1}^{n} \omega_{i}^{j}
$$

Be the $j^{\text {th }}$ breast sample moment, an estimate of $\mu_{j}$. The method of pixel points estimator for $\theta_{1}, \ldots, \theta_{k}$ denoted by $\check{\theta}_{1}, \ldots, \breve{\theta}^{k}$ is defined as the result to the equation is

$$
\begin{aligned}
\check{\mu}_{1} & =g_{1}\left(\check{\theta}_{1}, \ldots, \check{\theta}_{k}\right) \\
\check{\mu}_{2} & =g_{2}\left(\check{\theta}_{1}, \ldots, \check{\theta}_{k}\right) \\
\check{\mu}_{k} & =g_{k}\left(\check{\theta}_{1}, \ldots, \check{\theta}_{k}\right)
\end{aligned}
$$

Mean

The probability distribution of random pixels $X$ the mean is equal to sum over every possible pixel values.

$$
\mu=\sum x P(x)
$$

$\mathrm{x}$ denoted total number of pixels values of image.

An analogous formula applies to the case of a 2D Breast Pixels probability distribution. Not every 3D Breast pixels probability distribution has a defined mean. 


\section{Gradient Linear Mapping}
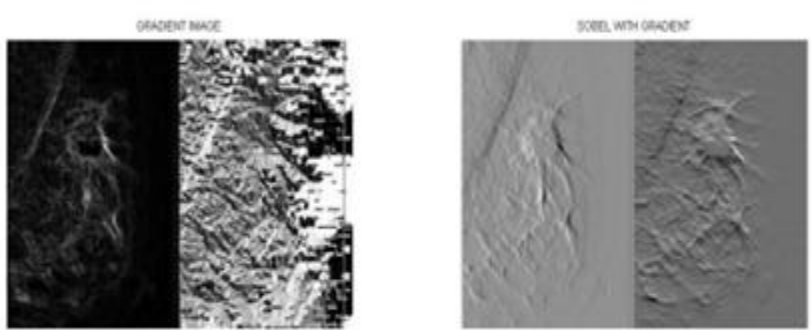

Figure 2: Gradient Linear Images

A Gradient Linear Mapping [10] algorithm performing a like as a affine Transform [6] of Euclidean pixels space which moves every pixel points by a fixed distance in the same direction in the breast images. The breast image every pixels are shifted the origin of coordinate[7] regions in the images.

$$
B_{r(i, j)}=p+v
$$

\section{FILTERING}

In this section we have used a nonlinear filtering. This filter used to reduce noise in the Breast data base. This process used to improve segmentation level. Mainly the median filter performing every pixels [includes rows and columns][9] are replaced by median pixel of the overall image.

$$
\begin{gathered}
\text { Bimg mid }=\frac{\text { pixels in } x}{\text { pixels in } y} \\
\operatorname{pixl}(x)=\frac{\text { pixel widt } h}{2} \\
\operatorname{pixel}(y)=\frac{\text { pixel hight }}{2}
\end{gathered}
$$

Above Equation (10) mention as Breast image median formula.

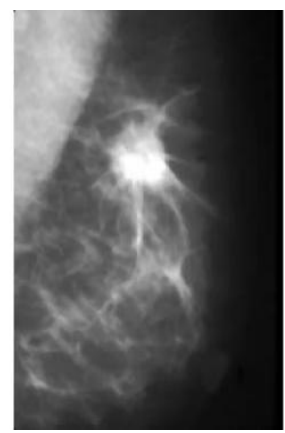

Figure 3: Median Filter Image

\section{ENHANCEMENT}

MCLAEH Enhancement algorithm is used in this section. This algorithm performing a histogram based enhances a breast images. So pixels are easily smoothed and then also to limiting a contrast level in every pixels.

This techniques mostly limiting contrast level in the image. So these techniques need some parameters from breast images. The parameters are mean of the image and then initial threshold values of image. The threshold image before apply mean selection of image. This technique also used to contrast level of the image. Contrast difference find to the original image. This technique helps to improve the transformation function of each pixel which is derived from a neighborhood breast region.

$$
T \text { - intensity thrshold value }
$$

$$
P_{T l}-\text { Pixel Intensity lower threshold value }
$$

$P_{\text {Th- }}$ Pixel Intensity Higher threshold

$$
\begin{aligned}
& \text { Mean }=\text { Tmean }(\text { Br Img }) \\
& \text { MCLAHE }=\frac{i m g-v_{-} m i n}{v_{\text {max }}-v_{-} m i n} \\
& \text { V. MORPHOLOGICAL OPERATION }
\end{aligned}
$$

\section{MORPHOLOGICAL OPERATION}

Morphological algorithm part of work is the Gray level Breast images are layer by layer analysis. The layers are extracted from Breast data's. the layers are Red layer, Green layer, Blue layer. All kind of this layer applied to image smoothing operation, then this details data's are applied to morphological operator like canny algorithm and also to enhance breast edge border to use Prewitt algorithm [11].

\section{Canny Algorithm Working}

1. Apply Gaussian Filter process

2. Find intensity gradients of the image

3. Apply suppression to image

4. Find potential edges

5. Extract edges from above steps

Gaussian Filter Equation

$$
G=\sigma^{2} * e(i-k-1)^{2}+(j-k-1)^{2}
$$

Gradient

$$
G=\operatorname{sqrt}\left(G^{2} x\right)+\left(G^{2} y\right)
$$
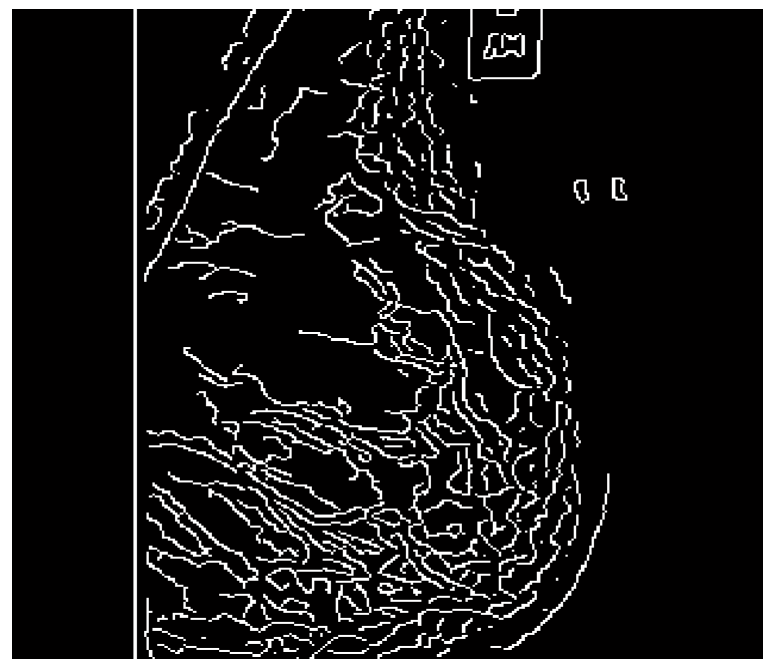

Figure 4: Canny Algorithm Image 


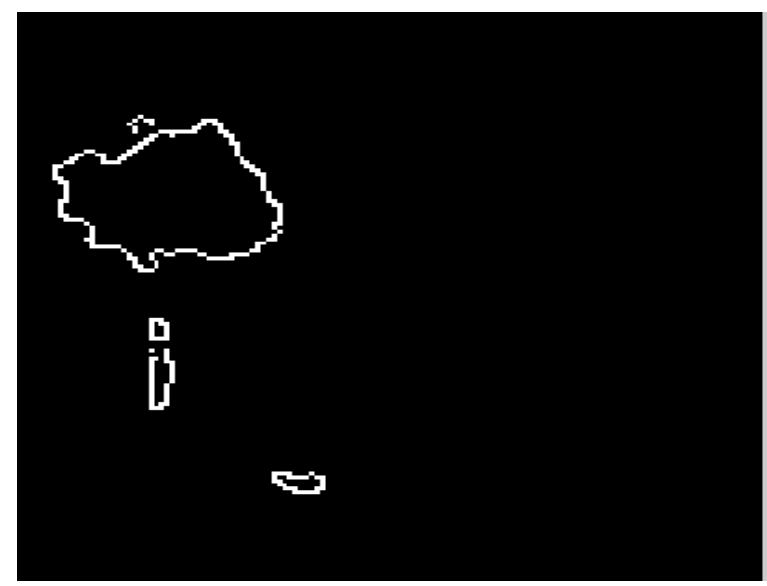

Figure 5: DL Outer Border Image

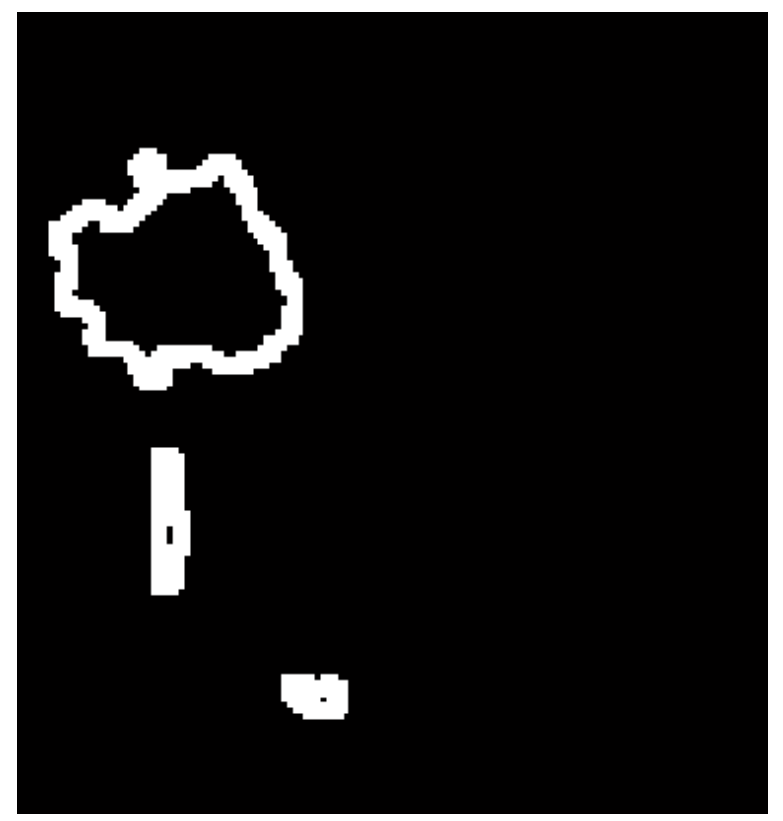

Figure 6: Dilated DL Image

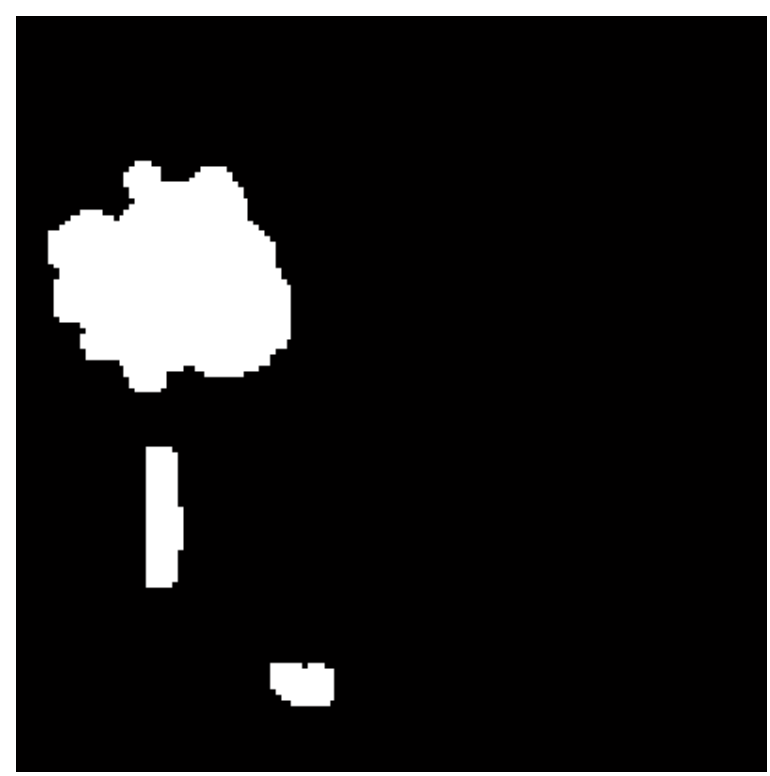

Figure 7: Breast Cancer Segment Region

\section{ANN CLASSIFICATION}

Artificial Neural Network based classification algorithms [8] are implemented to classify the Breast data base Classification [12] database are two types, Healthy Breast database and Unhealthy Breast database. Classification is mainly performs based on testing and training database features extraction process. The feature includes mean values of breast image, standard deviation of breast image, CNR of breast image, PSNR of breast image, and SSIM of breast images. This all kind of features extracted from both testing and also training database. The ANN algorithm background process is the based on features the database are classifying as class. The class like positive and negative element. This mathematical element based the database are classified.

Mean

$$
m=\frac{1}{m n} \sum_{i=0}^{m-1} \sum_{j=0}^{n-1}[I(i, j)-K(i, j)]^{2}
$$

PSNR

$$
\text { psnr }=10 . \log _{10} \frac{\max i^{2}}{m}
$$

CNR

$$
c n r=\frac{\left|S_{A}-S_{B}\right|}{\sigma_{o}}
$$

SSIM

$\operatorname{SSIM}(x, y)=\frac{\left(2 \mu_{x} \mu_{y}+C 1\right)\left(2 \sigma_{x y}+C 2\right)}{\left(\mu^{2}{ }_{x}+\mu^{2}{ }_{y}+C 1\right)\left(\sigma^{2}{ }_{x}+\sigma^{2}{ }_{y}+C 2\right)}$

$\mu_{x}$-average of $\mathrm{x}$

$\mu_{y}$-average of $\mathrm{y}$

$\sigma^{2}{ }_{x}$-variance of $\mathrm{x}$

$\sigma^{2}$-variance of $\mathrm{y}$

$\sigma_{x y}$-covariance of $\mathrm{x}$ and $\mathrm{y}$

$$
\begin{aligned}
& c_{1}=\left(k_{1} L\right)^{2} \\
& c_{2}=\left(k_{2} L\right)^{2}
\end{aligned}
$$

L- Dynamic range of the pixel value

$$
k_{1}=0.01 \text { and } k_{1}=0.03
$$

\section{DSSIM}

$$
\operatorname{DSSIM}(x, y)=\frac{1-\operatorname{ssim}(x, y)}{2}
$$

$\mathrm{x}$-pixel value of rows,

$y$-pixel value of columns. 
VII. AlgORITHM WORKING MODEL

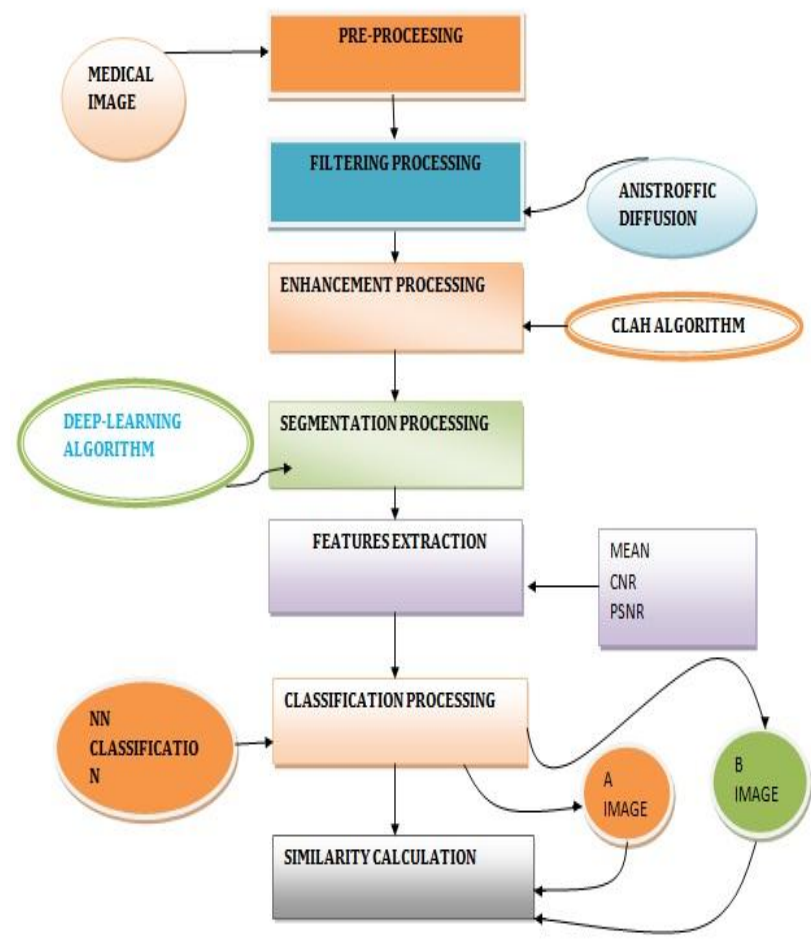

Figure 8: Algorithm for DL with ANN

VIII. OUtPut PeRformance

Input Breast Image

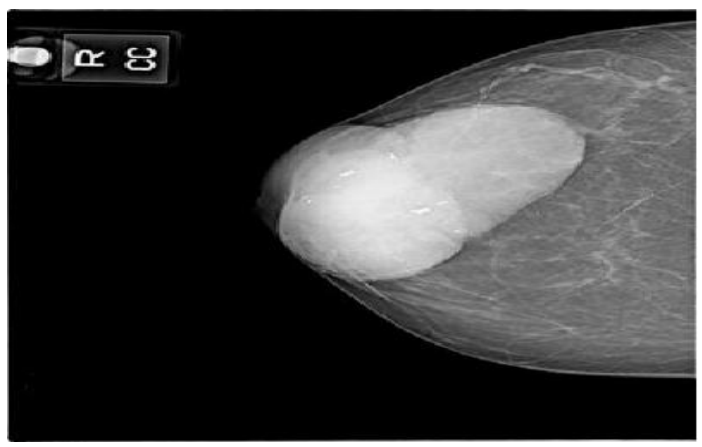

Figure 9: Input Breast Image

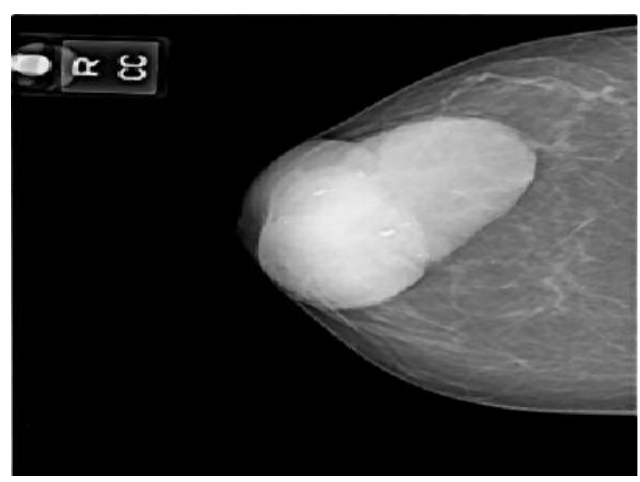

Figure 10: Filtered Image

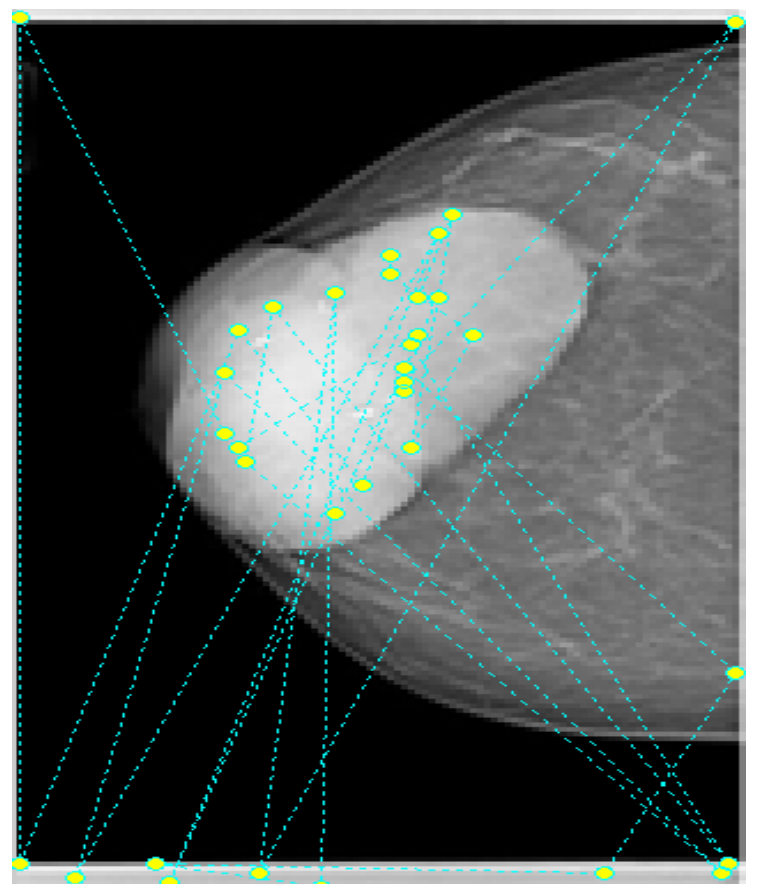

Figure 11: DL Mapping Image

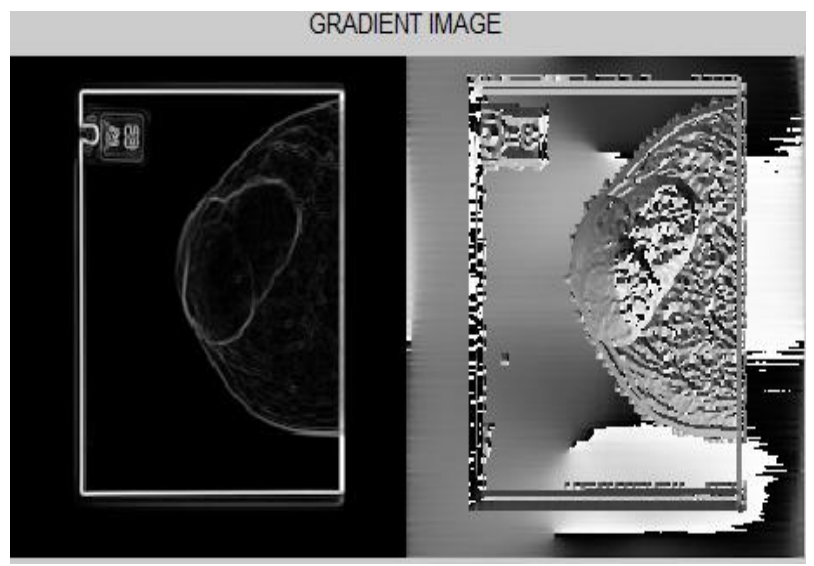

Figure 12: Gradient Image

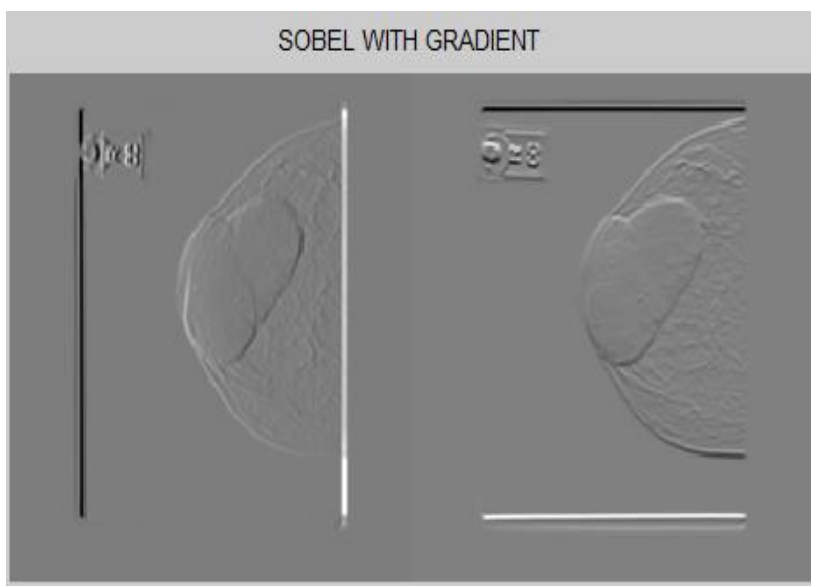

Figure 13: Sobel with Gradient Image 


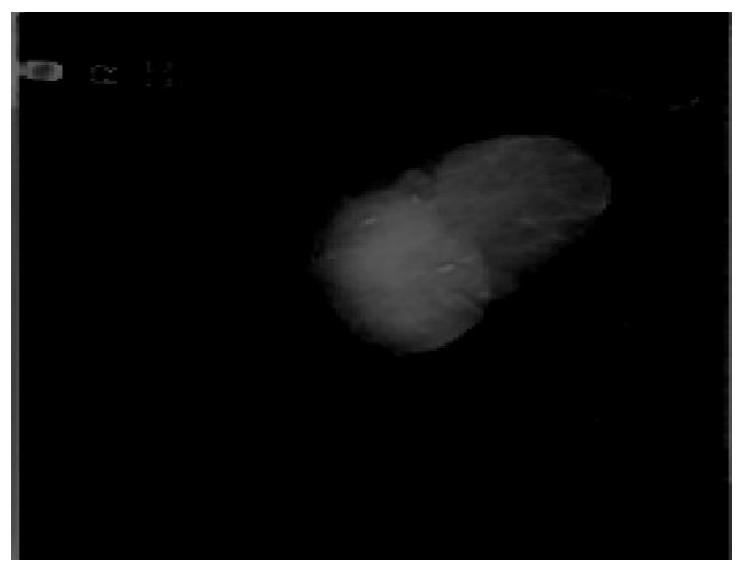

Figure 14: DL Masked Cancer Region

\section{DLL with Morphological Image}

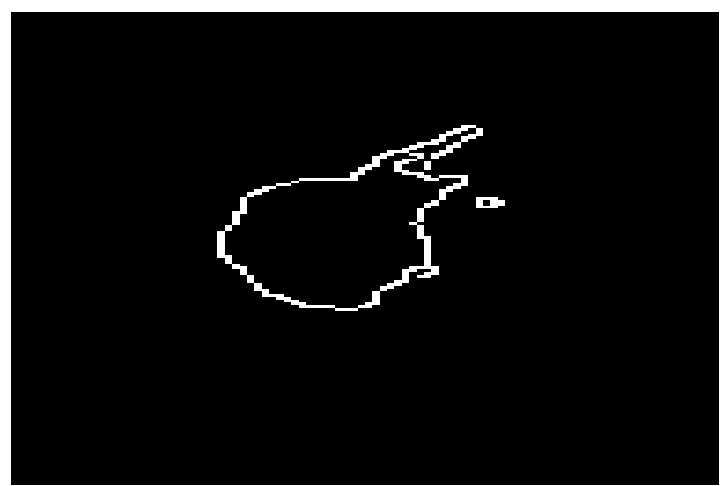

Figure 15: Binary Mask

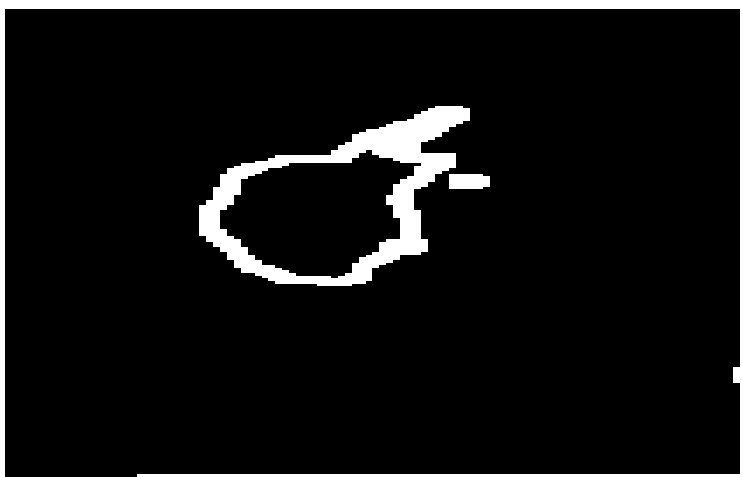

Figure 16: Dilated Binary Image

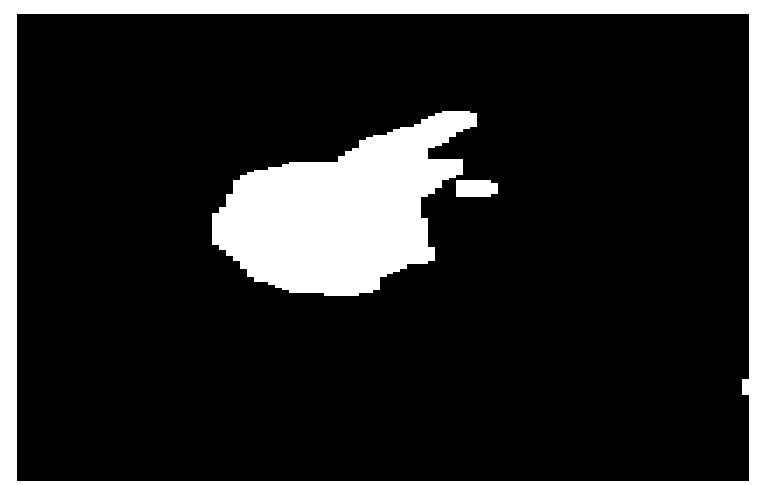

Figure 17: Holes Filled Image
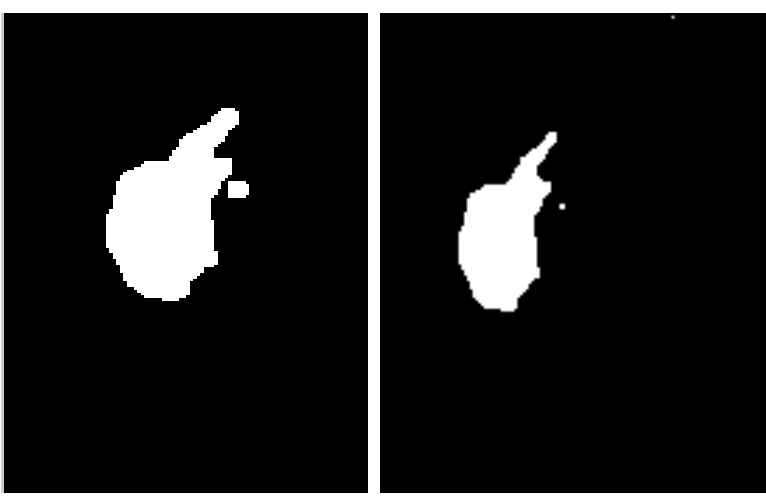

Figure 18: Breast Region

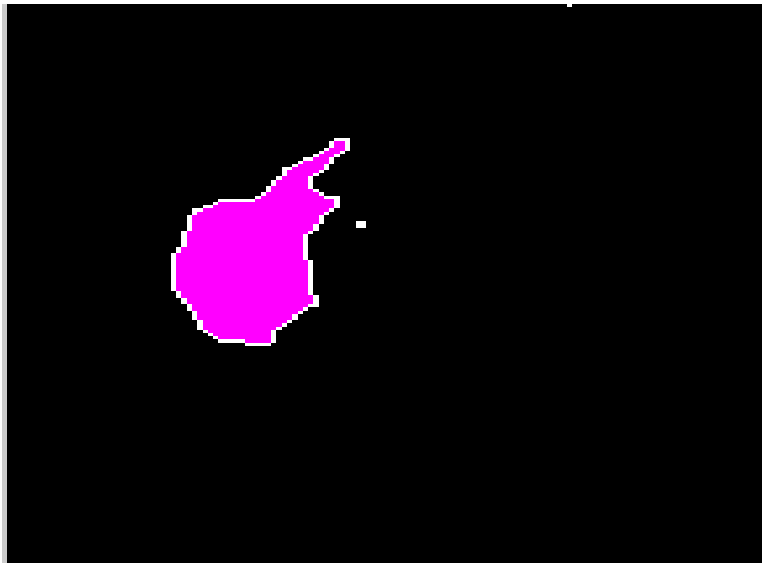

Figure 19: DL Breast Segment Region

\section{COMPARISON FEATURE PERFORMANCE}

Comparison performances of Breast segmentation feature algorithm are shown in table IX. This table is briefly explained about segmentation of breast cancer region. In this paper we have analysis breast image segmentation DL with NN algorithms are performed and it's tested from above tabulation. This tabulation mentioned as different features by breast images. They features are Peak to noise ratio(PSNR), Signal to noise ratio(SNR), Mean, Standard deviation (STD), Contrast to noise ratio(CNR), SSIM. This features peacefully extracted and they are performed. And also the variation is differentiated.

\section{Table 1: Comparison Feature Performance}

\begin{tabular}{|c|c|c|c|c|}
\hline Feature & SVM & $D L$ & $A N N$ & Proposed \\
\hline Mean & 12 & 11 & 7.5 & 7.56 \\
\hline PSNR & 1.0 & 1.2 & 1.55 & 2 \\
\hline STD & 5.2 & 4.2 & 5.3 & 4 \\
\hline CNR & 13 & 7 & 5 & 5.5 \\
\hline SSIM & 0.2 & 0.33 & 1.2 & 2.3 \\
\hline
\end{tabular}

\section{CONCLUSION}

In this paper, a segmentation approach is performed by DL with ANN segmentation algorithms. The segmentation algorithm mainly to segment breast images, and then extracting cancer region from breast images. The algorithm result shows that the various features from various algorithms and its all are parameters are evaluated. Mainly compared features by segmentation level algorithm. 


\section{REFERENCES}

[1] Arel I., Rose D.C., and Kamowski T.P., "Research frontier: Deep machine learning-a new frontier in artificial intelligence research," Comp. Intell Mag.5, 13-18(Nov 2010).

[2] Bengio Y., "Learning deep architectures for ai."Foundations and Trends in Machine Learning 2,1-127(Jan 2009).

[3] Bengio Y., Courville A., and Vincent P., "Representation learning: A review and new perspectives:, IEEE Transactions on Pattern Analysis and Machine Intelligence 35(8), 1798-1828(2013)

[4] Doyle S., Agner S.,Madabhushi A., Feldman M., and Tomaszewski J., "Automated grading of breast cancer histopathology using spectral clustering with textual and architectural image features", in [2008 $5^{\text {th }}$ IEEE International Symposium on Biomedical Imaging: From Nano to Macro]496-499, IEEE (May 2008)

[5] Elston C. and Ellis I., "Pathological prognostic factors in breast cancer the value of histological grade in breast cancer: experience from a large study with long term follow-up," Histopath 19(5), 403.410 (1991).

[6] Krizhevsky A., Sutskever I., and Hinton G.E., "Imagnet classification with deep convolutional neural networks", in [Advances in Neural Information Processing Systems 25], 1106-1114 (2012)

[7] Le Q., Han J.,Gray J., Spellman P., Borowsky A., and Parvin B., "Learning invariant features of tumor signatures" in [Biomedical Imaging (ISBI) $20129^{\text {th }}$ IEEE International Symposium on]302-305 (May 2012)

[8] Madabhushi A., "Digital pathology image analysis: opportunities and challenges (editorial)," Imaging in Medicine 1, 7-10 (October 2009)>

[9] Malon C., and Cosatto E., "Classification of mitotic figures with convolutional neural networks and seeded blob features", Journal of Pathology Informatics 4(1), 9(2013)

[10] Niwas S.I., Palanisamy P., Zhang W., Mat Isa N.A., and Chibbar R., "Log-gabor wavelets based breast carcinoma classification using least square support vector machine," in [2011 IEEE International Conference on Imaging Systems and Techniques], 219-223, IEEE (May 2011).

[11] Weston J., Bengio S., and Usunier N., "Large scale image annotation: Learning to rank with joint word image embeddings," Machine Learning 81, 21-35(Oct 2010).

Bandita Sen is currently pursuing her ME in Applied Electronics from Sathyabama University(2014-2016).She has finished her B.Tech in biomedical Engineering from West Bengal University of Technology. She has having industrial experience in different organization like Siemens, AMRI hospital (Kolkata),pro-interactive services. 